\title{
[actualización]
}

\section{Utilidad de la capilaroscopia en esclerodermia}

\author{
Génessis Maldonado Vélez ${ }^{1}$, Carlos Ríos Acosta²
}

${ }^{1}$ Médico General Universidad de Especialidades Espíritu Santo, Km. 2.5 Vía la Puntilla Samborondón-Ecuador. ${ }^{2}$ Médico Reumatólogo Centro de Reumatología y Rehabilitación, El Oro y Ambato 1004, Guayaquil-Ecuador

\section{Resumen}

La esclerosis sistémica (SSc) es una enfermedad del tejido conectivo caracterizado por una reactividad autoinmune, disfunción vascular generalizada y fibrosis progresiva de la piel y órganos internos asociado a la producción de anticuerpos específicos. Durante los últimos años, la capilaroscopia ha demostrado ser una herramienta útil, reproducible y confiable para la evaluación inicial del fenómeno de Raynaud y enfermedades del tejido conectivo, en especial de la esclerodermia, siendo esta técnica incluida en los criterios diagnósticos de la esclerodermia, lo que deja en evidencia la utilidad de esta técnica. A su vez la capilaroscopia asume un rol importante en la evaluación del compromiso sistémico, transición de la enfermedad, manejo y predictor de mortalidad de la esclerodermia, aspectos que serán discutidos en esta revisión.

Palabras clave: capilaroscopia del lecho ungueal, esclerodermia, patrón esclerodérmico (SD).

\section{Abstract}

Systemic sclerosis (SSc) is a connective tissue disease characterized by autoimmune reactivity, progressive generalized vascular dysfunction and fibrosis of the skin and internal organs associated with the production of specific antibodies. In recent years the nailfold capillaroscopy has proven to be an useful and realible tool for the initial evaluation of Raynaud's phenomenon and connective tissue diseases specially scleroderma, also this technique has been included in the diagnostic criteria of scleroderma, which demonstrate the usefulness of this technique. In turn, the capillaroscopy plays an important role in the evaluation of systemic involvement, transition of the disease, management and mortality predictor of scleroderma, aspects that will be discussed in this review.

Key words: nailfold capillaroscopy, scleroderma, SD Pattern.

\section{Introducción}

La esclerosis sistémica (SSc) es una enfermedad del tejido conectivo caracterizado por una reactividad autoinmune, disfunción vascular generalizada y fibrosis progresiva de la piel y órganos internos, lo que conlleva a producción de anticuerpos, microvasculopatías progresivas y depósito anormal de proteínas de matriz extracelular que afecta piel, pulmones, tracto gastrointestinal y daño de órganos severos ${ }^{1}$.

La incidencia anual global se estima que es de 19 casos por un millón y una prevalencia de $19-75$ por 100.000 habitantes's en el Caribe, la prevalencia de esclerodermia es de aproximadamente 9,3 por millón de habitantes ${ }^{3}$, siendo menor a la presentada en Argentina la cual es de 296 casos por un millón de habitantes y una incidencia de 6,1 por un millón de habitantes en un $\mathrm{año}^{4}$; además, la esclerodermia es más común en mujeres 3:1 frente a hombres².

Las manifestaciones clínicas de la esclerodermia se dividen en dos grandes grupos: la presencia de Fenómeno de Raynaud (FR) y compromiso cutáneo y una prevalencia variable de compromiso de órganos internos ${ }^{1}$. Scolnik y cols. describieron las manifestaciones clínicas de una cohorte de pacientes con esclerodermia en la que se evidenció dismotilidad esofágica 64,3\%, enfermedad intersticial difusa $65,3 \%$, hipertensión pulmonar aislada $2,6 \%$, crisis renales $0,02 \%$, úlceras digitales $32,1 \%{ }^{5}$. El compromiso cutáneo puede ser clasificado de acuerdo a la extensión: limitada (lcSSc) o difusa (dcSSc) ${ }^{2}$.

El fenómeno de Raynaud fue descrito por primera vez en la tesis de Maurice Raynaud en el año 1862, como isquemia local de manos, pies, nariz y lengua que puede estar acompañado de dolor ${ }^{6}$; en la actualidad se define como un trastorno isquémico episódico manifestado por palidez, cianosis y rubor de la piel en respuesta a estímulos como el frío o el estrés emocional'; puede ser primario o secundario a enfermedades del tejido conectivo en especial a la esclerodermia; representa el primer signo clínico de una alteración del tono vascular sistémico.

La capilaroscopia es un método no invasivo, seguro que permite la visualización de los capilares a nivel del lecho ungueal ${ }^{8}$; entre sus indicaciones se encuentran ${ }^{7}$ :

1. Evaluación de enfermedades vasculares periféricas, específicamente en la diferenciación del Fenómeno de Raynaud primario y secundario.

2. Evaluación del compromiso microvascular de las enfermedades del tejido conectivo como: esclerodermia, lupus, dermatomiositis y enfermedad mixta del tejido conectivo.

\section{Correspondencia}

E-mails: genesismaldonadovelez92@gmail.com - criosacosta@gmail.com 
Esta revisión destaca la importancia de la capilaroscopia en el estudio de la esclerodermia, además, de la inclusión de esta herramienta a los criterios diagnósticos de la enfermedad, predictor de transición, evaluación del compromiso sistémico y predictor de mortalidad.

\section{Capilaroscopia y esclerodermia}

La microangiopatía es un aspecto clínico patognomónico de la esclerodermia y la capilaroscopia permite identificar este daño mediante patrones morfológicos específicos en las diferentes fases de la enfermedad.

En el año 1981, Maricq describió un patrón capilaroscópico, llamado patrón esclerodérmico o SD, compuesto por':

- Capilares gigantes: según las descripciones de Maricq los capilares con un diámetro de asa apical de 25-50 $\mu \mathrm{m}$ se consideran dentro del rango normal, aquellos que sobrepasen el rango normal de 4-10 veces el ancho del asa apical ${ }^{10-12}$ se denominan capilares gigantes.

- Zonas avasculares: definidas como la ausencia de capilares por un tracto superior a $500 \mu \mathrm{m}^{13}$.

- Hemorragias: Depósito de hemosiderina, provocada por la ruptura de uno o varios capilares.

- Capilares arborificados: fueron descritos como aquellos capilares que adoptan la forma similar a un árbol ${ }^{13}$.

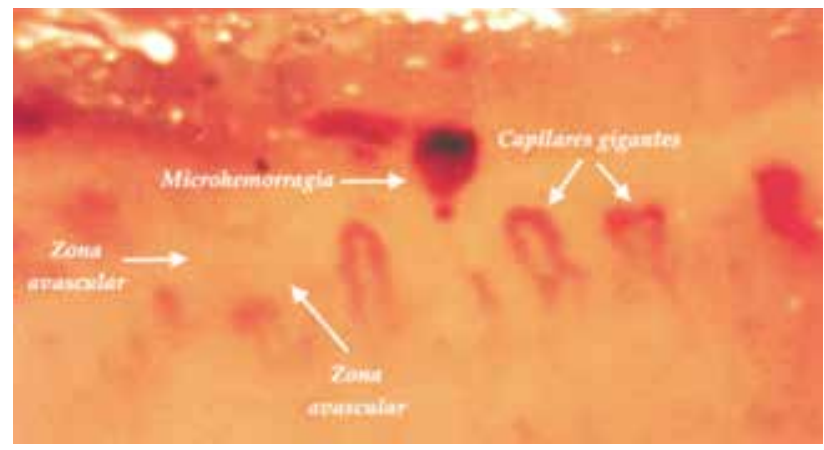

Figura 1. Patrón SD compuesto por: microhemorragias, capilares gigantes y zonas avasculares. Foto original tomada por los autores.

Maricq evidenció que el patrón SD está presente en un $83-98 \%$ de los pacientes con esclerodermia; además, los estudios realizados por Cutolo demuestran que la capilaroscopia del lecho ungueal presenta hallazgos indicativos de microangiopatía esclerodérmica en un 80 $90 \%$ de los casos de pacientes con esclerodermi ${ }^{14-16}$.

Cutolo describió una clasificación de alteraciones capilaroscópicas en base al patrón SD, en tres fases: temprana, activa y $\operatorname{tardía}^{14}$ (Tabla 1) (Figuras 2, 3, 4, 5 y 6).

\section{Escalas de medición en capilaroscopia}

La capilaroscopia puede ser evaluada por métodos cuantitativos o semicuantitativos.

\begin{tabular}{|c|c|c|c|c|}
\hline Fase & Distribución & $\begin{array}{c}\text { Capilares } \\
\text { gigantes }\end{array}$ & Hemorragias & $\begin{array}{c}\text { Pérdida } \\
\text { capilar }\end{array}$ \\
\hline Temprana & Preservada & + & + & - \\
\hline Activa & $\begin{array}{c}\text { Leve } \\
\text { desorganización }\end{array}$ & ++ & ++ & ++ \\
\hline Tardía & $\begin{array}{c}\text { Moderada } \\
\text { desorganización }\end{array}$ & + & + & +++ \\
\hline
\end{tabular}

Tabla 1. Alteraciones capilaroscópicas en fases de la esclerodermia.

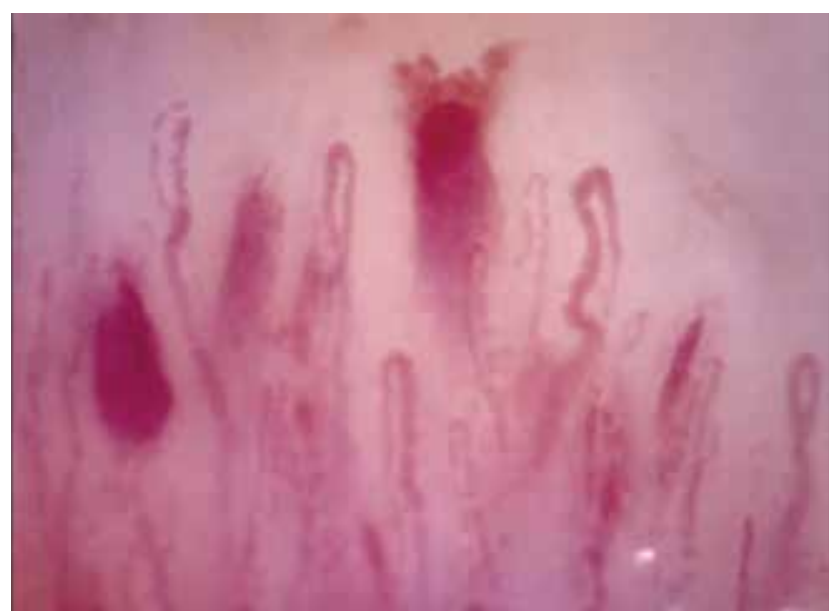

Figura 2. Patrón esclerodérmico temprano: Hemorragias, capilares dilatados, distribución capilar conservada. No hay evidencia de pérdida capilar. Foto original tomada por los autores.

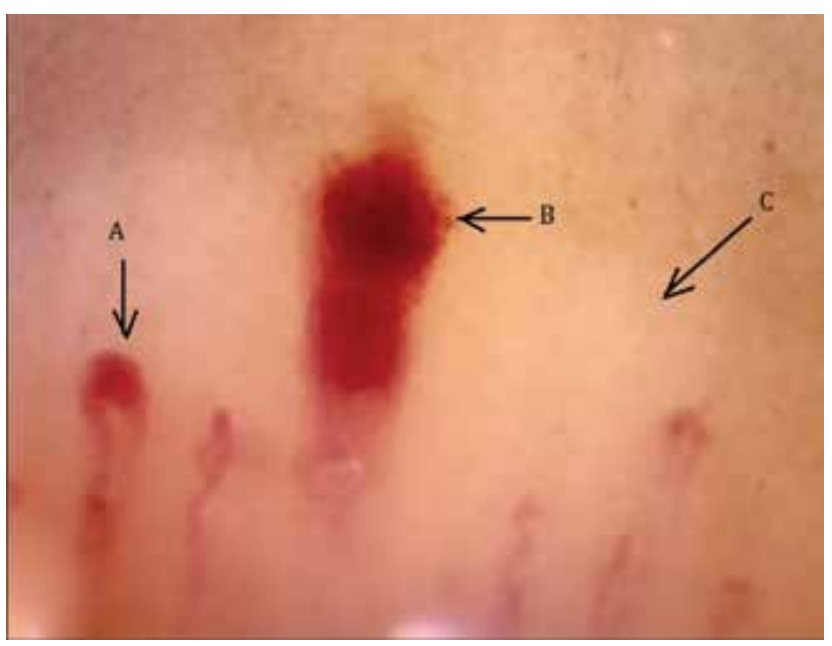

Figura 3. Patrón esclerodérmico activo: $\boldsymbol{A}$. Capilar dilatado, B. Microhemorragia, C. Zonas avasculares y reducción de la densidad capilar. Foto original tomada por los autores.

\section{Método cuantitativo:}

El método cuantitativo se basa en determinación de la densidad capilar (media de número de capilares), capilares gigantes, hemorragias, arborificaciones ${ }^{17}$. El proceso es el mismo para cada hallazgo capilaroscópico; a continuación será detallado el proceso de cada uno de ellos: 


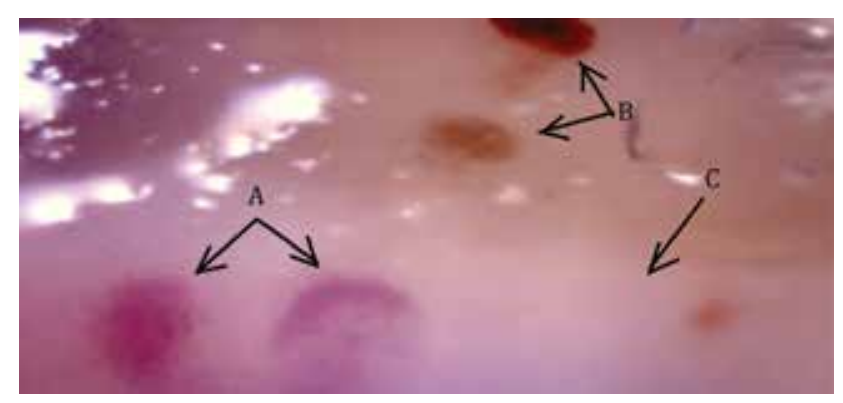

Figura 4. Patrón esclerodérmico activo: $\boldsymbol{A}$. Capilares gigantes, $\boldsymbol{B}$. Depósito de hemosiderina/hemorragias. C. Zona avascular. Foto original tomada por los autores.

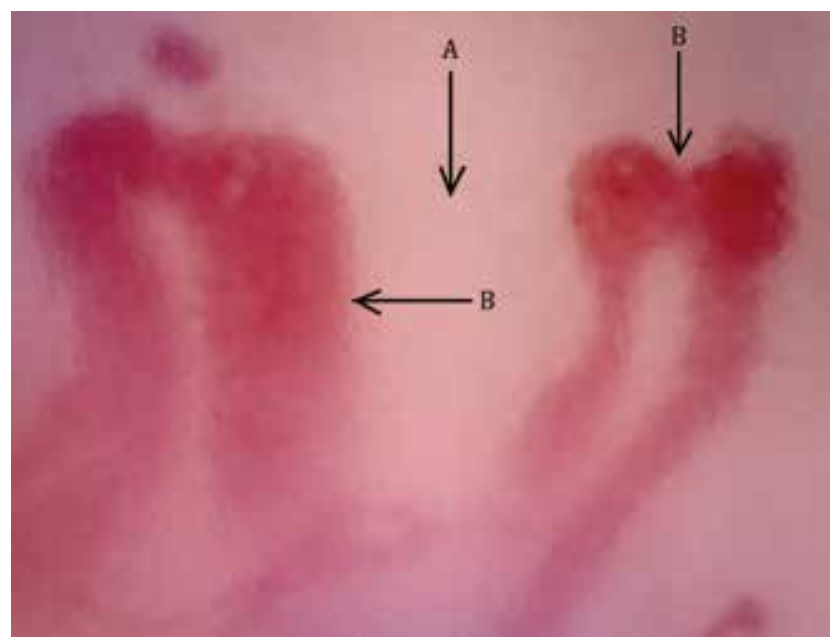

Figura 5. Patrón esclerodérmico tardío: A. Pérdida capilar y zonas avasculares, $\boldsymbol{B}$. Capilares gigantes. Foto original tomada por los autores.

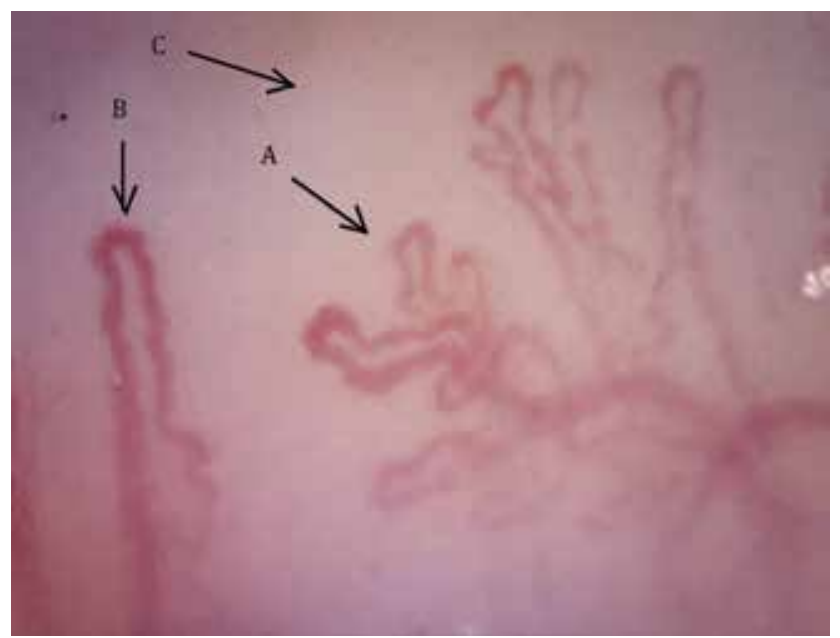

Figura 6. Patrón esclerodérmico tardío: $\boldsymbol{A}$. Capilar arborificado, B. Capilar dilatado, C. Zonas avasculares. Foto original tomada por los autores.
- Densidad capilar: La determinación de la densidad capilar se calcula al dividir para 8 la suma del número de capilares observados en $1 \mathrm{~mm}$ lineal de cada dedo examinado.

- Megacapilares: Se calcula mediante la suma de los megacapilares en un $1 \mathrm{~mm}$ de campo lineal de cada dedo examinado y el total se divide para 8.

- Hemorragias: Se calcula mediante la suma de las hemorragias encontradas en un $1 \mathrm{~mm}$ de campo lineal de los dedos examinados y el total se divide para 8.

- Arborificaciones: Se calcula al dividir para 8 el total de arborificaciones encontradas en los dedos examinados. Nota: La medición de los campos deben ser siempre de una zona central del lecho ungueal. Se cuentan los capilares de la primera fila. Se recomienda estudiar todos los dedos exceptuando los pulgares.

\section{Método semicuantitativo:}

Por otro lado el método semicuantitativo tiene un enfoque general y específico. Sulli propusó una escala general semicuantativa de las alteraciones capilaroscópicas (densidad capilar, megacapilares, hemorragias y arborificaciones) y les asignó un score del 0-3 ${ }^{18}$ (Tabla 2).

\begin{tabular}{|c|c|}
\hline Puntaje & Descripción \\
\hline 0 & Normal \\
\hline 1 & $<33 \%$ de los capilares observados en $1 \mathrm{~mm}$ lineal \\
\hline 2 & $33-66 \%$ de los capilares observados en $1 \mathrm{~mm}$ lineal \\
\hline 3 & $>66 \%$ de los capilares observados en $1 \mathrm{~mm}$ lineal \\
\hline
\end{tabular}

Tabla 2. Escala general semicuantitativa de las alteraciones capilaroscópicas.

\section{Escalas específicas de alteraciones capilaroscópicas}

Las escalas semicuantitativas evalúan: densidad capilar, megacapilares, hemorragias y arborificaciones.

- Densidad capilar: El score de la densidad capilar se determina asignando un score de la escala (Tabla 3) a cada dedo observado y el total de los dos dedos se divide para 8.

- Megacapilares: De manera similar se asigna un score a cada dedo de acuerdo a la escala (Tabla 4) y el total de las dos manos se dividen para 8.

- Hemorragias: Para la determinación de hemorragias se asigna un score de acuerdo a la escala (Tabla 5) y la suma total de los dedos observados se divide para 8.

- Arborificaciones: De manera similar se asigna un score de la escala semicuantitativa (Tabla 6) a cada dedo observado y la suma total es dividido para 8 .

Smith y cols. elaboraron una escala capilaroscópica como pronóstico de las lesiones tróficas, identificaron que la media de pérdida de la densidad capilar puede ser el parámetro fundamental para la evaluación de las lesiones tróficas, mediante la escala semicuantitativa de la densidad capilar (Tabla 2), se evidenció que aquellos pacientes con más lesiones tróficas cutáneas tenían scores semicuantitativos elevados entre 2-3, lo que hace esta técnica reproducible en la práctica clínica ${ }^{19}$. 


\begin{tabular}{|c|c|}
\hline Puntaje & Descripción \\
\hline 0 & $>9$ capilares $/ \mathrm{mm}$ lineal \\
\hline 1 & $7-9$ capilares $/ \mathrm{mm}$ lineal \\
\hline 2 & $4-6$ capilares $/ \mathrm{mm}$ lineal \\
\hline 3 & $1-3$ capilares $/ \mathrm{mm}$ lineal \\
\hline
\end{tabular}

Tabla 3. Escala semicuantitativa de la densidad capilar.

\begin{tabular}{|c|c|}
\hline Puntaje & Descripción \\
\hline 0 & Ausencia de megacapilares en $1 \mathrm{~mm}$ lineal \\
\hline 1 & $\begin{array}{r}\text { Presencia de megacapilares }<33 \% \text { de todos los } \\
\text { capilares observados en } 1 \mathrm{~mm} \text { lineal }\end{array}$ \\
\hline 2 & $\begin{array}{c}\text { Presencia de megacapilares entre } 33-66 \% \text { de todos los } \\
\text { capilares observados en } 1 \mathrm{~mm} \text { lineal }\end{array}$ \\
\hline 3 & $\begin{array}{c}\text { Presencia de megacapilares }>66 \% \text { de todos los capilares } \\
\text { observados en } 1 \mathrm{~mm} \text { lineal }\end{array}$ \\
\hline
\end{tabular}

Tabla 4. Escala semicuantitativa de megacapilares.

\begin{tabular}{|c|c|}
\hline Puntaje & Descripción \\
\hline 0 & Ausencia de hemorragias en $1 \mathrm{~mm}$ lineal \\
\hline 1 & $\begin{array}{c}\text { Presencia de hemorragias }<33 \% \text { de todos los } \\
\text { capilares observados en } 1 \mathrm{~mm} \text { lineal }\end{array}$ \\
\hline 2 & $\begin{array}{c}\text { Presencia de hemorragias entre } 33-66 \% \text { de todos los } \\
\text { capilares observados en } 1 \mathrm{~mm} \text { lineal }\end{array}$ \\
\hline 3 & $\begin{array}{c}\text { Presencia de hemorragias }>66 \% \text { de todos los capilares } \\
\text { observados en } 1 \mathrm{~mm} \text { lineal }\end{array}$ \\
\hline
\end{tabular}

Tabla 5. Escala semicuantitativa de hemorragias.

\begin{tabular}{|c|c|}
\hline Puntaje & Descripción \\
\hline 0 & Ausencia de arborificaciones en $1 \mathrm{~mm}$ lineal \\
\hline 1 & $\begin{array}{c}\text { Presencia de arborificaciones }<33 \% \text { de todos los capilares } \\
\text { observados en } 1 \mathrm{~mm} \text { lineal }\end{array}$ \\
\hline 2 & $\begin{array}{c}\text { Presencia de arborificaciones entre } 33-66 \% \text { de todos los capilares } \\
\text { observados en } 1 \mathrm{~mm} \text { lineal }\end{array}$ \\
\hline 3 & $\begin{array}{c}\text { Presencia de arborificaciones }>66 \% \text { de todos los capilares } \\
\text { observados en } 1 \mathrm{~mm} \text { lineal }\end{array}$ \\
\hline
\end{tabular}

Tabla 6. Escala semicuantitativa de arborificaciones.

\section{Criterios de clasificación de la esclerodermia: inclusión de la capilaroscopia}

Los criterios preliminares para la clasificación de la esclerodermia fueron publicados por Masi y cols. en el año 1980 (Tabla 7); estos incluían la presencia de esclerosis cutánea de zonas proximales a las articulaciones metacarpofalanges o metatarsofalanges como criterios mayores y la presencia de dos o tres criterios menores, esclerodactilia, cicatrices ungueales (pitting) y fibrosis pulmonar bibasilar ${ }^{20}$. Estos criterios permitían identificar a los pacientes con esclerodermia, sin embargo, varios reportes demostraron que estos criterios tenían baja sensibilidad en especial en el grupo de pacientes con esclerodermia limitada y detección tardía de compromiso sistémico ${ }^{21-23}$.

En 1988, Leroy y cols. publicaron los criterios de clasificación de esclerodermia; estos dividían a la esclerodermia en dos subtipos:

- Esclerodermia limitada: afectación de piel, principalmente cara, codos y rodillas.

- Esclerodermia difusa: afectación de piel incluyendo el tronco.

\begin{tabular}{|c|c|}
\hline Criterios & Descripción \\
\hline Criterios mayores & $\begin{array}{c}\text { Esclerosis de articulaciones proximales } \\
\text { (metacarpofalanges/metatarsofalanges) }\end{array}$ \\
\hline Criterios menores & Esclerodactilia \\
Úlceras digitales \\
Fibrosis pulmonar bibasilar
\end{tabular}

Se establece el diagnóstico con la presencia de un criterio mayor 0 al menos 203 criterios menores.

Tabla 7. Criterios de clasificación de Esclerosis Sistémica ACR $1980^{20}$.

Además utilizaron la capilaroscopia para el estudio del compromiso vascular y evidenciaron que los pacientes con esclerodermia tenían alteraciones capilaroscópicas específicas, motivo por el cual se decidió incluir a esta técnica en los criterios de clasificación, lo que aumentó significativamente la sensibilidad y especificidad de los mismos ${ }^{24}$.

En el año 2001, LeRoy y Medsger plantearon nuevo concepto de diagnóstico temprano de Esclerodermia, lo que generó un gran impacto en la detección temprana de pacientes con esclerodermia; estos criterios determinaban que incluso en ausencia de compromiso cutáneo, el diagnóstico de esclerodermia era posible con la presencia de fenómeno de Raynaud, capilaroscopia alterada y sugestiva de patrón SD y presencia de anticuerpos específicos ${ }^{25}$ (Tabla 8).

\begin{tabular}{|c|}
\hline \multicolumn{1}{|c|}{ Presencia de Fenómeno de Raynaud más: } \\
\hline Capilaroscopia anormal (megacapilares \pm zonas avasculares \\
\hline y/o presencia de: \\
\hline $\begin{array}{c}\text { Anticuerpos específicos (anticentrómero } 0 \text { anti-Scl70 o anti-RNApolimerasa } \\
\text { III } 0 \text { anti-PmSCl } 0 \text { anti-Th/To) }\end{array}$ \\
\hline
\end{tabular}

Tabla 8. Criterios de diagnóstico temprano de esclerodermia ${ }^{25}$.

No obstante, en el año 2009, el grupo EULAR Scleroderma Trial and Research group (EUSTAR) propusieron el término "diagnóstico muy temprano de esclerodermia (VEEDOS)"; basado en un consenso y opiniones de expertos crearon estos criterios que incluían: presencia de Fenómeno de Raynaud, anticuerpos específicos (anti-nucleares, anti-CENP, anti-topo I) y capilaroscopia anormal como criterios mayores y calcinosis, manos puffy, úlceras digitales, disfunción del esfínter gastroesofágico, telangiectasias y presencia de signo radiológico de vidrio esmerilado en tomografía de toráx como criterios adicionales; se consideraba el diagnóstico muy temprano de Esclerodermia si cumplía al menos tres criterios mayores o dos criterios mayores acompañados de un criterio adicional ${ }^{26}$.

La última actualización de los criterios fue realizada por la ACR/EULAR en el 2013, la cual presentó una sensibilidad del $91 \%$ y especificidad del $92 \%{ }^{27}$ (Tabla 9 ).

\section{Predictor de transición de la enfermedad}

Uno de los estudios más importantes en relación con los predictores de la enfermedad realizado por Koenig, se 


\begin{tabular}{|c|c|c|}
\hline Item & Sub-item & Score \\
\hline $\begin{array}{c}\text { Engrosamiento de la piel } \\
\text { en ambas manos que se } \\
\text { extiende a metacarpofalanges } \\
\text { (criterio suficiente) }\end{array}$ & - & 9 \\
\hline $\begin{array}{c}\text { Engrosamiento de los dedos } \\
\text { (solo cuenta el score más alto) }\end{array}$ & & \\
\hline Lesiones digitales & Manos puffy & 2 \\
\hline (solo cuenta el score más alto) & Úlceras digitales & 2 \\
\hline Telangiectasias & Pitting ungueal & 3 \\
\hline Capilaroscopia anormal & - & 2 \\
\hline Hipertensión pulmonar y/0 & Hipertensión pulm. & 2 \\
\cline { 2 - 3 } enfermedad pulmonar intersticial & Enfermedad pulmonar & 4 \\
\hline intersticial & 2 \\
\hline Fenómeno de Raynaud & - & 3 \\
\hline Anticuerpos & Anti-centrómero & 3 \\
& Anti-Scl-70 & \\
\hline
\end{tabular}

Se considera esclerodermia a un score mayor 0 igual a 9 .

Tabla 9. Criterios diagnósticos 2013 ACR/EULAR para esclerodermia.

basó en capilaroscopias y determinación de anticuerpos anti-centrómero, anti-Th/To, anti-topoisomerasa I y antiRNA polimerasa III en pacientes referidos a un centro de reumatología para la evaluación del fenómeno de Raynaud, y encontró que una capilaroscopia anormal y la presencia de anticuerpos lleva a la aparición de esclerodermia de $47 \%$ a los 5 años, $72,7 \%$ a los 10 años y 79,5\% a los 15 años. La presencia de anti-CENP-B y anti-Th/To se relacionan con capilares dilatados y los Anti-RNAP III con zonas avasculares; el resultado de este estudio demostró que la incidencia de progresión de fenómeno de Raynaud a esclerodermia fue del $12,6 \%$ similar a los resultados mostrados por Spencer-Green ${ }^{28,29}$.

Sulli y cols. estudiaron el tiempo de transición de la enfermedad mediante identificación de patrones capilaroscópicos en pacientes con esclerosis sistémica, la media del tiempo de progresión de un patrón esclerodérmico temprano a activo fue de $28 \pm 20$ meses y de un patrón activo a tardío de $36 \pm 29$ meses, además de identificar un subgrupo de pacientes con una progresión rápida de microangiopatía ( $\leq 1$ año), aproximadamente el $50 \%$ de los pacientes con esclerodermia tenían daño microvascular con una transición dinámica dentro de los diferentes patrones capilaroscópicos; concluyen que se deben hacer controles capilaroscópicos cada seis meses. Además, se evidenció una progresión de los signos y síntomas de acuerdo a los cambios capilaroscópicos, se encontró una relación directa entre la microangiopatía de fase tardía y mayor compromiso sistémico; aquellos pacientes con un patrón tardío presentaron compromiso esofágico, pulmonar, úlceras digitales, hipertensión pulmonar, esclerodactilia y compromiso cutáneo limitado y difuso ${ }^{30}$.

\section{Evaluación del compromiso sistémico}

Los patrones capilaroscópicos en esclerodermia se asocian con la gravedad de la enfermedad y la afectación multisistémica, es por esto que la capilaroscopia ayuda al estadiaje del paciente y proporciona información pronóstica ${ }^{31}$.

Riccieri investigó cambios capilaroscópicos específicos en pacientes con esclerodermia con y sin hipertensión pulmonar y observó que los pacientes con hipertensión pulmonar tenían más zonas avasculares $(83 \%)$ frente aquellos que no lo tenían (17\%). Se vio un patrón activo/ tardío mayor en pacientes con hipertensión pulmonar en un $92 \%$ y al comparar la media de presión arterial pulmonar y las zonas avasculares se evidenció una relación significativa ${ }^{32}$.

Caramaschi estudió la asociación entre la capilaroscopia y manifestaciones clínicas en pacientes con esclerodermia difusa y limitada; demostró que los cambios capilaroscópicos se correlacionan con el subconjunto de la enfermedad, gravedad vascular periférica, compromiso cutáneo, cardíaco y pulmonar ${ }^{31}$.

En cuanto al compromiso pulmonar, Matucci-Cerinic observó una fuerte correlación entre las alteraciones capilaroscópicas y la capacidad de difusión del monóxido de carbono (DLCO), ya sea por la presencia de enfermedad pulmonar intersticial o hipertensión pulmonar; además la reducción de DLCO podría ser indicativo de microangiopatía pulmonar ${ }^{33}$.

La pérdida capilar está relacionada con hipertensión arterial pulmonar, enfermedad intersticial, severidad de la enfermedad vascular periférica y compromiso pulmonar o cardíaco según la escala de Medsger ${ }^{34}$.

Un estudio realizado por Sambarato demostró que las microhemorragias y los capilares gigantes son indicadores de actividad de la enfermedad en pacientes con esclerodermia y que las microhemorragias se relacionan con un compromiso cutáneo severo y manifestaciones clínicas cardiopulmonares ${ }^{35}$.

En el 2013, Khanna publicó recomendaciones para la evaluación de pacientes con enfermedades reumáticas y sugiere que los pacientes con presencia de patrón SD deberían someterse a un screening anual para el diagnóstico de hipertensión pulmonar ${ }^{36}$.

Las úlceras digitales son una manifestación frecuente de microangiopatía en pacientes con esclerodermia. Sebastani utilizó la capilaroscopia para la evaluación temprana de las úlceras en 120 pacientes, aproximadamente el 29,2\% desarrolló úlceras digitales y estas se asociaron a presencia de capilares gigantes, tortuosos y microhemorragias ${ }^{37}$.

Sebastiani realizó un índice de riesgo de desarrollo de úlceras digitales (CSURI) con una sensibilidad del $94,3 \%$ y especificidad del $85,9 \%$ con un valor predictivo positivo de 6,68 y negativo de 0,07 . Este índice se basa en la cuantificación y relación con el número de capilares de la última fila distal $(\mathrm{N})$, número de capilares gigantes $(\mathrm{M})$ y la presencia o ausencia de tortuosidades y microhemorragias; con un punto de corte de 2,94, aquellos valores por encima de ese valor tienen mayor probabilidad de desarrollar úlceras digitales siendo una nueva herramienta predictiva del desarrollo de úlceras digitales en pacientes con esclerodermia ${ }^{37}$.

Morardet y cols. determinaron la relación entre la presencia de calcinosis y acro-osteolisis en pacientes con esclerosis sistémica y las alteraciones capilaroscópicas; aquellos pacientes con acro-osteolisis y calcinosis tenían un patrón SD tardío, caracterizado por pérdida severa de 
la densidad capilar y presencia de neoangiogenesis, se cree que se deba a un intento de compensar la resorción ósea. Este fue el primer estudio que demostró esta asociación.

Cutolo y cols. realizaron el primer estudio multicéntrico prospectivo, llamado CAP Study, en el cual evaluaron las características clínicas y capilaroscópicas de pacientes con esclerosis sistémica e identificaron los factores de riesgo para la aparición de nuevas úlceras digitales en un período de 6 meses; aquellos pacientes con historia de úlceras digitales presentaron nuevas úlceras digitales en un $22 \%$ durante el seguimiento frente al grupo sin historia de úlceras digitales, en el cual solo el $5 \%$ desarrollaron úlceras digitales. Además, los pacientes con antecedentes de úlceras digitales presentaron un patrón capilaroscópico tardío y una diminución de la densidad capilar. Dentro de los factores de riesgo para la nueva aparición de úlceras digitales se destacó: la media de la densidad capilar en el dedo anular de la mano dominante, el número de úlceras digitales identificadas al momento de ingresar al estudio y la isquemia digital ${ }^{38}$.

\section{Capilaroscopia y anticuerpos}

Koenig realizó capilaroscopia y determinación de anticuerpos anti-centrómero, anti-Th/To, antitopoisomerasa I y anti-RNA polimerasa III en pacientes referidos a un centro de reumatología para evaluación de Fenómeno de Raynaud y demostró que una capilaroscopia anormal y la presencia de anticuerpos lleva a una aparición de Esclerodermia en $47 \%$ en 5 años, $69 \%$ en 10 años y $79 \%$ en 15 años, la presencia de anti-CENP-B y anti-Th/To se relacionan con capilares dilatados y Anti-RNAP III con zonas avasculares; el resultado de este estudio demostró que la incidencia de progresión de Fenómeno de Raynaud a esclerodermia fue del $12,6 \%{ }^{28}$.

En otro estudio, Cutolo usó solamente la capilaroscopia ( $\sin$ detección de anticuerpos) en pacientes con Fenómeno de Raynaud y observó que la transición a una colagenopatía se vio en un $15 \%$ de pacientes ${ }^{39}$.

\section{Predictor de mortalidad}

Kayser y cols. determinaron que la presencia de zonas avasculares y capilares gigantes en pacientes con esclerodermia son factores independientes de mortalidad; a su vez, aquellos pacientes con un score de zonas avasculares $\geq 1,5$ tienen dos veces más riesgo de mortalidad que aquellos con valores inferiores ${ }^{40}$.

\section{Conclusiones}

La capilaroscopia es una técnica no invasiva, reproducible que permite el estudio de la microarquitectura a nivel del lecho periungueal. Su uso se ha incrementado en los últimos años y ha demostrado ser una herramienta de alto valor predictivo negativo para el diagnóstico de enfermedades del tejido conectivo.

Se ha demostrado que la capilaroscopia es el mejor método validado para la evaluación de la microarquitectura capilar en pacientes con esclerodermia; debido a esto, a lo largo de los años ha sido incluida en los criterios de clasificación: ACR/EULAR 2013, criterios de clasificación de esclerodermia precoz de Leroy y criterios de diagnóstico muy temprano VEEDOS ${ }^{25,26,41}$.

Durante los últimos años se ha evidenciado un gran progreso del manejo de la esclerodermia, destacando una conducta de diagnóstico precoz que permite disminuir la morbimortalidad de estos pacientes. Es importante destacar que la capilaroscopia forma parte del manejo de esta enfermedad aportando datos claves y decisivos en la práctica clínica.

\section{Conflictos de intereses}

No existen conflictos de intereses.

\section{Bibliografía}

1. Cutolo M, Sulli A, Pizzorni C, Paolino S, Smith V. Systemic sclerosis: markers and targeted treatments. Acta Reum Port. 2016;41:18-25.

2. Gabrielli A, Avvedimento E, Krieg T. Scleroderma. N Eng J Med. 2009;360:1989-2003.

3. Gottschalk P, Vásquez R, López P, Then J, Tineo C, Loyo E. Esclerodermia en el Caribe: características en una serie de casos dominicana. Reum Clin. 2014;10(6):373-9.

4. Rosa J, Soriano E, Narvaez-Ponce L, Del Cid C, Imamura P, Cataggio L. Incidence and prevalence of systemic sclerosis in a healthcare plan in Buenos Aires. J Clin Rheumatol. 2011;17:59-63.

5. Scolnik M, Lancioni E, Saucedo C, Marin J, Sabelli M, Bedran Z, et al. Systemic sclerosis in Argentina: evaluation of a large cohort from a single centre and comparison with other international series. Clin Exp Rheumatol. 2014; 32(6 Suppl 86):S-94-7.

6. Raynaud M. Thése de Médicine: De l'asphyxie locale et de la gangrene des extremités. Paris: Leclere. 1862;

7. Ríos C, Maldonado G. Manual de Capilaroscopia [Internet]. Primera Ed. Guayaquil: Centro de Reumatología y Rehabilitación; 2016. 103 p. Available from: http://panlar.org/es/recursos_post_type/ manual-de-capilaroscopia/

8. Maricq H, Leroy E, D’Angelo W. Diagnostic potential of in vivo capillary microscopy in scleroderma and related disorders. Arthritis Rheum. 1980;23:183-9.

9. Maricq H. Widefield capillary microscopy technique and Rating Scale for abnormalities seen in Scleroderma and Related Disorders. Arthritis Rheum. 1981;9:1159-65.

10. Hou M, Huang S, Wang C, Tseng L Lo, Chen Y. A computerized system of nail-fold capillaroscopy for dry eye disease diagnosis. Multidimens Syst Signal Process. 2012;23(4):512-24.

11. Lefford F, Edwards J. Nailfold capillary microscopy in connective tissue disease: quantitative morphological analysis. Ann Rheum Dis. 1986;45:741-9.

12. Hofstee H, Serné E, Roberts C. A multicentre study on the reliability of qualitative and quantitative nailfold videocapillaroscopy assessment. Rheumatology. 2012;51(4):749-55. 
13. Grassi W, Del Medico P. Atlas of Capillaroscopy. Milan: Edra Medical Publishing \& New Media; 2004.

14. Cutolo M, Sulli A, Pizzorni C, Accardo S. Nailfold videocapillaroscopy assessment of microvascular damage in systemic sclerosis. J Rheumatol. 2000;27(155):60.

15. Cutolo M, Grassi W, Cerinic MM. Raynaud's Phenomenon and the Role of Capillaroscopy. 2003;48(11):3023-30.

16. Cutolo M, Pizzorni C, Sulli A. Capillaroscopy. Best Pr Res Clin Rheumatol. 2005;3(437-52).

17. GREC - Grupo de Trabajo para el Estudio de la Capilaroscopia. Capilaroscopia. España; 2015.

18. Sulli A, Sechi M, Pizzorni C, Cutolo M. Scoring the nailfold microvascular changes during the capillaroscopic analysis in systemic sclerosis patients. Ann Rheum Dis. 2008;67:885-7.

19. Smith V, De Keyser F, Pizzorni C, Van Praet J, Decuman S, Sulli A, et al. Nailfold capillaroscopy for day-to-day clinical use: construction of a simple scoring modality as a clinical prognostic index for digital trophic lesions. Ann Rheum Dis. 2011;70:180-3.

20. Masi A, Rodnan G, Medsger JT, Altman R, D’Angelo W, Fries J. Preliminary criteria for the classification of systemic sclerosis (scleroderma). Arthritis Rheum. 1980;23:581-90.

21. Lonzetti L, Joyal F, Raynauld J. Updating the American College of Rheumatology preliminary classification criteria for systemic sclerosis: addition of severe nailfold capillaroscopy abnormalities makedly increases the sensitivity for limited scleroderma. Arthritis Rheum. 2001;44(735):6.

22. Hudson M, Taillefer S, Steele R, Dunne J, Johnson S, Jones N. Improving the sensitivity of the American College of Rheumatology classification criteria for systemic sclerosis. Clin Exp Rheumatol. 2007;25:754-7.

23. Ziswiler H, Urech R, Balmer J, Ostensen M, Mierau R, Villiger P. Clinical diagnosis compared to classification criteria in in a cohort of 54 patients with systemic sclerosis and associated disorders. Swiss Med Wkly. 2007;137:586-90.

24. LeRoy E, Black C, Fleismajer R, Jabolonska S, Krieg T, Medsger T. Scleroderma (systemic sclerosis): classification, subsets and pathogenesis. J Rheumatol. 1988;15:202-5.

25. LeRoy E, Medsger TJ. Criteria for the classification of early systemic sclerosis. J Rheumatol. 2001;28(1573):6.

26. Avouac J, Fransen J, Walker U. Preliminary criteria for the very early diagnosis of systemic sclerosis: results of a Delphi Consensus Study from EULAR Scleroderma Trials and Research Group. Ann Rheum Dis. 2011;70:476-81.

27. Hoogen V den. Classification Criteria for Sistemic Sclerosis. Arthritis Rheum. 2013;65:2737-47.

28. Koenig M, Joyal F, Fritzler MJ, Abrahamowicz M, Grodzicky T, Raymond Y, et al. Autoantibodies and Microvascular Damage Are Independent Predictive Factors for the Progression of Raynaud 's Phenomenon to Systemic Sclerosis A Twenty-Year Prospective Study of 586 Patients, With Validation of Proposed Criteria for Early Systemic Sclero. 2008;58(12):3902-12.

29. Spencer-Green G. Outcomes in primary Raynaud phenomenon: a metanalysis of the frequency, rates, and predictors of transition to secondary diseases. Arch Intern Med. 1998;(595-600).

30. Sulli A, Pizzorni C, Smith V, Zampogna G, Ravera F, Cutolo M. Timing of Transition Between Capillaroscopic Patterns in Systemic Sclerosis. 2012;64(3):821-5.

31. Caramaschi P, Canestrini S, Martinelli N, Volpe A, Pieropan S, Ferrari M, et al. Scleroderma patients nailfold videocapillaroscopic patterns are associated with disease subset and disease severity. Rheumatology. 2007;46:1566-9.

32. Riccieri V, Vasile M, Iannace N, Stefanantoni K, Sciarra I, Vizza C, et al. Systemic sclerosis patients with and without pulmonary arterial hypertension: a nailfold capillaroscopy study. Rheumatology (Oxford). 2013;52 (8)(1525):8.

33. Matucci-Cerinic M, D'Angelo S, Denton C, Vlachoyannopoulos P, Silver R. Assessment of lung involvement. Clin Exp Rheumatol. 2003;21(29):19-23.

34. Medsger TJ, Bombardieri S, Czirjak L, Scorza R, Della Rossa A, Bencivelli W. Assessment of disease severity and prognosis. Clin Exp Rheumatol. 2013;21(29):42-6.

35. Sambarato D, Sambarato G, Zaccara E, Maglione W, Polosa R, Afeltra A, et al. Nailfold videocapillaroscopy micro-haemorrhage and giant capillary counting as an accurate approach for a steady state definition of disease activity in systemic sclerosis. Arthritis Res Ther. 2014;16(5):462.

36. Khanna D, Gladue H, Channick R, Chung L, Distler O, Furst D. Recommendations for screening and detection of connective tissue disease-associated pulmonary arterial hypertension. Arthritis Rheum. 2013;65(3194):201.

37. Sebastiani M, Manfredi A, Colaci M, D’Amico D, Malagoli V, Giuggioli D, et al. Capillaroscopic Skin Ulcer Risk Index: A New Prognostic Tool for Digital Skin Ulcer Development in Systemic Sclerosis Patients. Arthritis Rheum. 2009;61(5):688-94.

38. Cutolo M, Herrick AL, Distler O, Becker M, Beltran E, Carpentier P, et al. Nailfold Videocapillaroscopic Features and Other Clinical Risk Factors for Digital Ulcers in Systemic Sclerosis A Multicenter, Prospective Cohort Study. Arthritis Rheumatol. 2016;68(10):2527-39.

39. Cutolo M, Sulli A, Sechi M, Olivieri M, Pizzorni C. The contribution of capillaroscopy to the differential diagnosis of connective autoimmune diseases. Best $\mathrm{Pr}$ Res Clin Rheumatol. 2007;21:1093-108.

40. Kayser C, Sekiyama J, Próspero L, Camargo C, Andrade L. Nailfold capillaroscopy abnormalities as predictors of mortality in patients with systemic sclerosis. Clin Exp Rheumatol. 2013;31(76):103-8.

41. Van den Hoogen F, Khanna D, Fransen J, Johnson SR, Baron M, Tyndall A, et al. 2013 classification criteria for systemic sclerosis: an American college of rheumatology/European league against rheumatism collaborative initiative. Ann Rheum Dis. 2013 Nov 1;72(11):1747-55. 